\title{
Linear independence of values of a certain generalisation of the exponential function - a new proof of a theorem of Carlson
}

\author{
par ROLF WALLISSER
}

RÉSumÉ. Soit $Q$ un polynôme non-constant à coefficients entiers, sans racines sur les nombres entiers positifs. Nous donnons ici, essentiellement avec la méthode de Hermite, une nouvelle démonstration de l'indépendence linéaire de certaines valeurs aux points rationnels de la fonction

$$
G(x)=\sum_{n=0}^{\infty} \frac{x^{n}}{Q(1) Q(2) \cdots Q(n)} .
$$

ABstract. Let $Q$ be a nonconstant polynomial with integer coefficients and without zeros at the non-negative integers. Essentially with the method of Hermite, a new proof is given on linear independence of values at rational points of the function

$$
G(x)=\sum_{n=0}^{\infty} \frac{x^{n}}{Q(1) Q(2) \cdots Q(n)} .
$$

\section{Introduction}

Let $Q \in \mathbb{Z}[x]$ be a nonconstant polynomial of degree $q$ with integer coefficients and without zeros at the non-negative integers. Carlson [2] investigated already in 1935 the arithmetical nature of values of the function

(1) $\quad G(x)=\sum_{n=0}^{\infty} \frac{x^{n}}{Q(1) Q(2) \cdots Q(n)}=1+\sum_{n=1}^{\infty} \frac{x^{n}}{Q(1) Q(2) \cdots Q(n)}$;

For $n=0$ we define $Q(1) \ldots Q(n)$ to be equal to one.

Carlson proves:

Theorem 1. Let $\alpha_{1}, \ldots, \alpha_{h}$ be any pairwise distinct non-zero rational numbers. Then we have for the function $G$ (compare (1)) that the values

$$
1, G\left(\alpha_{1}\right), \ldots, G^{(q-1)}\left(\alpha_{1}\right), \ldots, G\left(\alpha_{h}\right), \ldots, G^{(q-1)}\left(\alpha_{h}\right)
$$

are linearly independent over the field $\mathbb{Q}$ of rational numbers. 


\section{Remarks.}

i) $G$ satisfies a linear differential equation of order $q$ with coefficients which are polynomials over $\mathbb{Q}$. Therefore it is not possible to replace in the theorem the order $(q-1)$ of differentation by a higher one.

ii) One can assume that the numbers $\alpha_{j}(1 \leq j \leq h)$ in the theorem are integers. To show this, let us denote

$$
\begin{aligned}
\alpha_{j} & :=\frac{s_{j}}{t_{j}}(1 \leq j \leq h), s_{j}, t_{j} \in \mathbb{Z}^{*}, a:=t_{1} \cdot t_{2} \ldots t_{h}, \\
t_{j} \alpha_{j}^{*} & :=s_{j} a, \alpha_{j}^{*} \in \mathbb{Z}, Q^{*}(x):=a \cdot Q(x) .
\end{aligned}
$$

If the theorem is proved for integers $\alpha_{j}$ we use this result for the function

$$
\begin{gathered}
G^{*}(x)=\sum_{n=0}^{\infty} \frac{1}{Q(1) \cdots Q(n)}\left(\frac{x}{a}\right)^{n}=\sum_{n=0}^{\infty} \frac{x^{n}}{Q^{*}(1) \cdots Q^{*}(n)} ; \\
\text { If }\left(s_{0}, s_{1}, \ldots, s_{h}\right) \in \mathbb{Z}^{h+1} \backslash \overrightarrow{0} \text { it follows } \\
s_{0}+s_{1} G^{*}\left(\alpha_{1}^{*}\right)+\cdots+s_{h} G^{*}\left(\alpha_{h}^{*}\right) \neq 0
\end{gathered}
$$

The term on the left in this relation is by definition of $G^{*}$ and $\alpha_{j}^{*}:=$ $\alpha_{j} \cdot a$ equal to

$$
s_{0}+s_{1} G\left(\alpha_{1}\right)+\cdots+s_{h} G\left(\alpha_{h}\right)
$$

Carlson's proof depends on a certain Padé-approximation of $G$, a representation of the remainder term by an integral and a careful evaluation of this term using a Laurent development about infinity. Applying the method of Siegel-Shidlowskij similar results, qualitative and good quantitative ones, were obtained mainly by the Russian school (compare [4], pp. 128-136).

In this paper we use in principle the same method which Hermite applied to establish the transcendence of the basis of the natural logarithm. Like Hilbert [7] and Hurwitz [8] we apply divisibility properties to show the non-vanishing of a certain sum of integers. Aside the exponential function, this way was mainly used by Skolem [12] to get results on the irrationality of certain numbers. We take the method of Hermite in the form as it is described in Perron [10] and Skolem [12], and regain in a simple way the result of Carlson.

\section{The method of Hilbert-Perron-Skolem}

Let

$$
f(x)=\sum_{n=0}^{\infty} k_{n} x^{n}, k_{0}=1
$$


One chooses a "starting-polynomial"

$$
P(x):=P_{0}(x):=\sum_{n=0}^{m} \gamma_{n} k_{n} x^{n}
$$

and derives from $P \quad m$ other polynomials:

$$
P_{\mu}(x)=\sum_{n=\mu}^{m} \gamma_{n} k_{n-\mu} x^{n-\mu}, 1 \leq \mu \leq m .
$$

The "approximating-polynomial" $P^{*}$ belonging to $P$ is the following:

$$
P^{*}(x):=\sum_{\mu=0}^{m} P_{\mu}(x) .
$$

Remark. If $f$ denotes the exponential function (e.g $Q(x)=x$ or $\left.k_{n}=\frac{1}{n !}\right)$ one has the relation

$$
P^{*}(x)=\sum_{\mu=0}^{m} P^{(\mu)}(x) .
$$

Using the relations (4) and (5) one obtains

$$
P^{*}(x)=\sum_{n=0}^{m} \gamma_{n} \sum_{\nu=0}^{n} k_{\nu} x^{\nu}
$$

and with $f(0)=k_{0}=1$

$$
P^{*}(0)=\sum_{n=0}^{m} \gamma_{n} .
$$

Finally it follows for the "remainder-term"

$$
\Delta(x):=P^{*}(x)-P^{*}(0) f(x)
$$

with (7) and (8)

$$
\begin{aligned}
\Delta(x) & =\sum_{n=0}^{m} \gamma_{n} \sum_{\nu=0}^{n} k_{\nu} x^{\nu}-\sum_{n=0}^{m} \gamma_{n} \sum_{\nu=0}^{\infty} k_{\nu} x^{\nu} \\
& =-\sum_{n=0}^{m} \gamma_{n} \sum_{\nu=n+1}^{\infty} k_{\nu} x^{\nu} .
\end{aligned}
$$

To prove theorem 1 one has to show that for every non-zero vector

$$
\left(s_{0}, s_{1}^{(0)}, \ldots, s_{h}^{(0)}, \ldots, s_{1}^{(q-1)}, \ldots, s_{h}^{(q-1)}\right) \in \mathbb{Z}^{h q+1}
$$

the linear form

$$
\Lambda=s_{0} \cdot 1+\sum_{j=1}^{h} \sum_{\nu=0}^{q-1} s_{j}^{(\nu)} G^{(\nu)}\left(\alpha_{j}\right)
$$


does not vanish.

To show this one uses the "approximating-polynomial" $P^{*}$ (compare (6)) to introduce the linear form

$$
\Lambda^{*}=s_{0} P^{*}(0)+\sum_{j=1}^{h} \sum_{\nu=0}^{q-1} s_{j}^{(\nu)} P^{*(\nu)}\left(\alpha_{j}\right) .
$$

Using the "remainder term" $\Delta(x)$ (compare (9)) one gets the following connection between the linear forms $\Lambda$ and $\Lambda^{*}$

$$
\Lambda^{*}=P^{*}(0) \Lambda+\sum_{\nu=0}^{q-1} \sum_{j=1}^{h} s_{j}^{(\nu)} \Delta^{(\nu)}\left(\alpha_{j}\right) .
$$

If one chooses an appropriate "starting-polynomial" $P$ one can show firstly that the linear form $\Lambda^{*}$ is different from zero and secondly that

$$
\left|\sum_{\nu=0}^{q-1} \sum_{j=1}^{h} s_{j}^{(\nu)} \Delta^{(\nu)}\left(\alpha_{j}\right)\right| \leq \frac{1}{2}\left|\Lambda^{*}\right| .
$$

In this way one gets from (14)

$$
\left|P^{*}(0)\right| \cdot|\Lambda| \geq \frac{1}{2}\left|\Lambda^{*}\right|>0 .
$$

Form this inequaliy one concludes $\Lambda \neq 0$ which proves theorem 1 .

Remark. In the paper of Skolem [12] and in [1] one finds further applications of the method of Hilbert-Perron-Skolem.

\section{The form of the polynomials $P_{\mu}$ in the special case of the function $G$ defined in 1.}

We apply now the method of Hilbert-Perron-Skolem described in Chap 2 to the function $G$ defined in Chap 1. In this case the values $k_{n}$ in (3) are given by

$$
k_{n}:=\frac{1}{Q(1) Q(2) \ldots Q(n)} .
$$

Let $\delta$ denote the operator $x \frac{d}{d x}$. The function $G$ (compare (1)) satisfies the linear differential equation of order $q$, (compare [4], Chap 2, 6.1. formula (85)).

$$
Q(\delta) G(x)=Q(0)+x G(x) .
$$


If one applies $Q(\delta)$ to $P_{\mu}$ one gets

$$
\begin{aligned}
Q(\delta) P_{\mu}(x) & =\sum_{n=\mu}^{m} \gamma_{n} k_{n-\mu} Q(\delta) x^{n-\mu} \\
& =\sum_{n=\mu}^{m} \gamma_{n} k_{n-\mu} Q(n-\mu) x^{n-\mu} \\
& =\gamma_{\mu} Q(0)+x \sum_{n=\mu+1}^{m} \gamma_{n} k_{n-(\mu+1)} x^{n-(\mu+1)} \\
& =\gamma_{\mu} Q(0)+x P_{\mu+1}(x), 0 \leq \mu \leq m .
\end{aligned}
$$

If the "starting-polynomial" $P_{0}(x):=P(x)$ vanishes at zero with multiplicity $m_{0}$, it follows

$$
P_{\mu}(0)=\gamma_{\mu}=0 \quad \text { for } \quad \mu=0, \ldots, m_{0}-1
$$

and from (19) we have

$$
P_{\mu}(x)=\left(\frac{1}{x} Q(\delta)\right)^{\mu} \circ P(x) \quad \text { for } \quad 0 \leq \mu \leq m_{0} .
$$

At the end of this chapter we will prove a connection of the operators $\delta$ and $D=\frac{d}{d x}$ giving with (21) the following representation of the polynomials $P_{\mu}$,

$$
P_{\mu}(x)=\sum_{j=0}^{\mu \cdot q} \alpha_{j, \mu} x^{j-\mu} P^{(j)}(x), \mu \leq m_{0} .
$$

The coefficients $\alpha_{j, \mu}$ are integers and if $Q \in \mathbb{Z}[x]$ has the form

$$
Q(x)=a_{0}+a_{1} x+\cdots+a_{q} x^{q}, a_{q} \neq 0, q \geq 1
$$

one gets for the highest coefficient of $P_{\mu}$

$$
\alpha_{\mu q, \mu}=a_{q}^{\mu} .
$$

Lemma 1. For $\delta=x \frac{d}{d x}$ and $D=\frac{d}{d x}$ we have

$$
\begin{aligned}
\delta^{j} & =\sum_{i=0}^{j} \rho_{i, j} x^{i} D^{i}, \rho_{i j} \in \mathbb{Z}, \\
\rho_{0,0} & :=1 ; \rho_{0, j}:=0, \rho_{i, j}:=i \rho_{i, j-1}+\rho_{i-1, j-1}(0<i<j), \\
\rho_{j, j} & :=\rho_{j-1, j-1}(0<j), \rho_{i, j}:=0(i=-1 \text { or } i>j) .
\end{aligned}
$$


Beweis. The lemma holds for $j=0$. Assume that the lemma has been verified for $j=0,1, \ldots, k$. From this inductive hypothesis one deduces:

$$
\begin{aligned}
\delta^{k+1} f(x) & =\left(x \frac{d}{d x}\right)^{k+1}(f(x))=\left(x \frac{d}{d x}\right) \sum_{i=0}^{k} \rho_{i, k} x^{i} f^{(i)}(x) \\
& =\sum_{i=0}^{k} i \rho_{i, k} x^{i} f^{(i)}(x)+\sum_{i=0}^{k} \rho_{i, k} x^{i+1} f^{(i+1)}(x) \\
& =\sum_{i=0}^{k+1}\left(i \rho_{i, k}+\rho_{i-1, k}\right) x^{i} f^{(i)}(x) \\
& =\sum_{i=0}^{k+1} \rho_{i, k+1} x^{i} D^{i} f(x) .
\end{aligned}
$$

The lemma follows by induction.

Lemma 2. For $Q(x)=\sum_{\ell=0}^{q} a_{\ell} x^{\ell}$ one has

i) $Q(\delta)=\sum_{j=0}^{q} \alpha_{j} x^{j} D^{j}, \alpha_{j}:=\sum_{\ell=j}^{q} \rho_{j, \ell} a_{\ell}(0 \leq j \leq q)$

ii) $\left(\frac{1}{x} Q(\delta)\right)^{\mu}=\sum_{j=0}^{\mu \cdot q} \alpha_{j, \mu} x^{j-\mu} D^{j}, \alpha_{0,0}:=1, \alpha_{j, 1}:=\alpha_{j}(0 \leq j \leq q)$

$$
\alpha_{\mu q, \mu}=\alpha_{q, 1}^{\mu}=\alpha_{q}^{\mu}=\left(\rho_{q, q} a_{q}\right)^{\mu}=a_{q}^{\mu}
$$

Beweis. From Lemma 1 we gain

$$
\begin{aligned}
Q(\delta) & =\sum_{\ell=0}^{q} a_{\ell} \delta^{\ell}=\sum_{\ell=0}^{q} a_{\ell}\left(\sum_{i=0}^{\ell} \rho_{i, \ell} x^{i} D^{i}\right) \\
& =\sum_{j=0}^{q} x^{j} D^{j}\left(\sum_{\ell=j}^{q} \rho_{j, \ell} a_{\ell}\right)=\sum_{j=0}^{q} \alpha_{j} x^{j} D^{j} .
\end{aligned}
$$

(ii) We use induction. Clearly the formula holds for $\mu=0$. Assume that ii) is proved for $\mu=0,1, \ldots, k$. For the following it is not necessary to have an explicit expression for the numbers $\alpha_{j, \mu}$ for $j=0, \ldots, q \mu-1, \mu \geq 1$. 
We get

$$
\begin{aligned}
\left(\frac{1}{x} Q(\delta)\right)^{k+1}= & \sum_{i=0}^{q} \alpha_{i, 1} x^{i-1} \sum_{j=0}^{q \cdot k} \alpha_{j, k} D^{i}\left(x^{j-k} D^{j}\right) \\
= & \sum_{i=0}^{q} \alpha_{i, 1} x^{i-1} \sum_{j=0}^{q \cdot k} \alpha_{j, k} \\
& \times \sum_{h=0}^{i}\left(\begin{array}{c}
i \\
h
\end{array}\right)(j-k) \ldots(j-k-h+1) x^{j-k-h} D^{i+j-h} \\
= & \sum_{i=0}^{q} \sum_{j=0}^{q \cdot k} \sum_{h=0}^{i} \alpha_{i, 1} \alpha_{j, k}\left(\begin{array}{c}
i \\
h
\end{array}\right)\left(\begin{array}{c}
j-k \\
h
\end{array}\right) h ! x^{i+j-h-(k+1)} D^{i+j-h} \\
= & \sum_{r=0}^{q \cdot(k+1)} \alpha_{r, k+1} x^{r-(k+1)} D^{r} .
\end{aligned}
$$

To finish the proof, we see

$$
\alpha_{q(k+1), k+1}=\alpha_{q, 1} \alpha_{q \cdot k, k}=\left(a_{q} \cdot a_{q}^{k}\right)=a_{q}^{k+1} .
$$

\section{A survey of some results on higher congruences}

Let $\mathbb{P}$ denote the set of prime numbers. For the proof of theorem 1 one needs some results on those primes $p \in \mathbb{P}$ for which the congruence $f(x) \equiv 0(\bmod p)$ is solvable. Here $f(x)$ denotes a polynomial with rational integer coefficients which is not identically zero $(\bmod p)$. Moreover results on those primes $p$ are needed for which the given polynomial splits mod $p$ in degree of $f(\operatorname{deg} f)$ linear factors. In algebraic-number-theory one can find many papers in this direction going essentially back to Dedekind [3] and Hasse [6]. Gerst and Brillhart [5] have given an excellent more elementary introduction to these problems. For better readability of this work I mention here some notations, definitions, conclusions and theorems. All proofs of these results can be found in [5].

Definition 1. A prime $p$ for which $f(x)$ is not identically zero $(\bmod p)$ and for which the congruence $f(x) \equiv 0(\bmod p)$ is solvable is called a prime divisor of $f$.

Proposition 1. Schur [11] has shown that every nonconstant polynomial $f$ has an infinite number of prime divisors. 
Definition 2. Let $f(x)=a \prod f_{i}(x)^{\beta_{i}}\left(a, \beta_{i} \in \mathbb{Z}, \beta_{i}>0, f_{i}(x) \in \mathbb{Z}[x]\right.$ distinct, primitive and irreducible) be the unique factorisation of $f(x)$ into irreducible polynomials. $f(x)$ will be said to "split completely" $(\bmod p), p$ a prime, iff each $f_{i}(x)$ is congruent $(\bmod p)$ to a product of $\operatorname{deg} f_{i}$ distinct linear factors and $p$ doesn't divide the discriminant of $g(x)=\prod f_{i}(x)$.

If $f(x)$ "splits completely" $(\bmod p)$ then $f(x)$ is congruent $(\bmod p)$ to a product of $\operatorname{deg} f$ linear factors of the form $a x+b, a, b \in \mathbb{Z}, p \nmid a$. It is this property which is needed in our prove of theorem 1.

Theorem 5 of the work of Gerst and Brillhart [5] gives a general information on the prime divisors of a polynomial. The following Proposition 2 is proved there as a corollary (compare [5], page 258).

Proposition 2. Every non-constant polynomial $f(x)$ has an infinite number of prime divisors $p$ for which it "splits completely" $(\bmod p)$.

\section{The choice of the "starting-polynomial" $P$ and some divisibility properties of the values $P_{\mu}^{(\rho)}\left(\alpha_{j}\right)$}

To prove theorem 1 one can assume that in (11) or (12) not all of the numbers $s_{j}^{(\nu)}(1 \leq j \leq h, 0 \leq \nu \leq q-1)$ are zero.

Let the value $s_{j_{0}}^{\left(\nu_{0}\right)}$ be the "highest" term in (11) which is different from zero; that is:

$$
\begin{aligned}
& s_{j_{0}}^{\left(\nu_{0}\right)} \neq 0, \\
& s_{j}^{(\nu)}=0, \quad 1 \leq j \leq h, \quad \nu_{0}<\nu \leq q-1, \\
& s_{j}^{\left(\nu_{0}\right)}=0, \quad j_{0}<j \leq h .
\end{aligned}
$$

So in case (25) the linear form $\Lambda^{*}$ of $(13)$ has the form

$$
\Lambda^{*}=s_{0} P^{*}(0)+\sum_{j=1}^{h} \sum_{\nu=0}^{\nu_{0}-1} s_{j}^{(\nu)} P^{*(\nu)}\left(\alpha_{j}\right)+\sum_{j=1}^{j_{0}} s_{j}^{\left(\nu_{0}\right)} P^{*\left(\nu_{0}\right)}\left(\alpha_{j}\right) .
$$

Let $p \in \mathbb{P}$ be a prime number which satisfies the relation:

$$
Q \text { "splits completely" }(\bmod p)
$$

(compare Definition 2 and Proposition 2 in Chap 4). Choose a sufficiently large $p$ with (26) which fulfills in addition the conditions

$$
p \nmid s_{j_{0}}^{\left(\nu_{0}\right)}, p \nmid a_{q} \alpha_{j_{0}} \prod_{\substack{j=1 \\ j \neq j_{0}}}^{h}\left(\alpha_{j_{0}}-\alpha_{j}\right) .
$$


Define the "starting-polynomial" $P$ of the method of Hilbert-PerronSkolem (compare Chap 2, (4)) in the following way:

$$
P(x):=x^{p \cdot q}\left(x-\alpha_{j_{0}}\right)^{(p-1) q+\nu_{0}} \prod_{\substack{j=1 \\ j \neq j_{0}}}^{h}\left(x-\alpha_{j}\right)^{p \cdot q} .
$$

Then the derivatives of $P$ satisfy the relations:

i) $P^{(\ell)}\left(\alpha_{j_{0}}\right)=\left\{\begin{array}{cc}0, & \ell<(p-1) q+\nu_{0}, \\ \alpha_{j_{0}}^{p \cdot q}\left((p-1) q+\nu_{0}\right) ! \times & \\ \prod_{\substack{j=1 \\ j \neq j_{0}}}^{h}\left(\alpha_{j_{0}}-\alpha_{j}\right)^{p \cdot q}, & \ell=(p-1) q+\nu_{0} .\end{array}\right.$

ii) $1 \leq j \leq h, j \neq j_{0}$,

$$
\begin{aligned}
& P^{(\ell)}\left(\alpha_{j}\right)= \begin{cases}0, & \ell<p \cdot q, \\
(q \cdot p) ! \alpha_{j}^{p \cdot q}\left(\alpha_{j}-\alpha_{j_{0}}\right)^{(p-1) q+\nu_{0}} \times & \\
\prod_{\substack{r=1 \\
r \neq j_{0}, j}}^{h}\left(\alpha_{j}-\alpha_{r}\right)^{p \cdot q}, & \ell=p \cdot q .\end{cases} \\
& \text { iii) } P^{(\ell)}(0)= \begin{cases}0, & \ell<p \cdot q, \\
(p q) !\left(-\alpha_{j_{0}}\right)^{(p-1) q+\nu_{0}} \prod_{\substack{j=1 \\
j \neq j_{0}}}^{h}\left(-\alpha_{j}\right)^{p \cdot q}, & \ell=p \cdot q .\end{cases}
\end{aligned}
$$

The Taylor development at $x=0$ of the "starting-polynomial" $P$ begins with the term $c x^{p \cdot q}, c \neq 0$. Therefore one concludes from (20)

$$
P_{\mu}(0)=0 \text { for } \mu=0, \ldots, p \cdot q-1 .
$$

From (22) one gets for $\mu \leq p \cdot q$

$$
P_{\mu}^{(\rho)}(x)=\sum_{j=0}^{\mu \cdot q} \alpha_{j, \mu}\left(x^{j-\mu} P^{(j)}(x)\right)^{(\rho)} .
$$

and from this follows with (29) for $\mu<p$

$$
P_{\mu}^{(\rho)}\left(\alpha_{j_{0}}\right)= \begin{cases}0 & 0 \leq \mu<p-1,0 \leq \rho \leq q-1, \\ 0 & \text { for } \quad \mu=p-1,0 \leq \rho<\nu_{0}, \\ A_{\nu_{0}} & \mu=p-1, \rho=\nu_{0} .\end{cases}
$$

$$
\begin{aligned}
A_{\nu_{0}} & :=\left((p-1) q+\nu_{0}\right) !\left(\alpha_{j_{0}} \prod_{\substack{j=1 \\
j \neq j_{0}}}^{h}\left(\alpha_{j_{0}}-\alpha_{j}\right)\right)^{p \cdot q} \alpha_{j_{0}}^{(p-1)(q-1)} \cdot \alpha_{(p-1) q, p-1}, \\
a_{q}^{p-1} & =\alpha_{(p-1) q, p-1} .
\end{aligned}
$$


From (27), (32) and (33) one concludes

$$
p^{q} \backslash P_{p-1}^{\left(\nu_{0}\right)}\left(\alpha_{j_{0}}\right), q \geq 1
$$

With (29) and (31) one gets (the same holds for $\alpha_{0}:=0$ ),

$$
P_{\mu}^{(\rho)}\left(\alpha_{j}\right)=0, j \neq j_{0}, \mu \leq p-1, \rho \leq q-1 .
$$

To find such properties for $\mu \geq p$, one takes the representation which follows from (5)

$$
P_{\mu}^{(\rho)}\left(\alpha_{j}\right)=\sum_{n=\mu+\rho}^{m} \gamma_{n} k_{n-\mu} \rho !\left(\begin{array}{c}
n-\mu \\
\rho
\end{array}\right) \alpha_{j}^{n-(\mu+\rho)}
$$

and one expresses $\gamma_{n} \cdot k_{n-\mu}$ (compare the definition of $k_{n}$ in (17)) in the following way:

$$
\gamma_{n} \cdot k_{n-\mu}=\gamma_{n} \cdot k_{n} \frac{k_{n-\mu}}{k_{n}}=\gamma_{n} \cdot k_{n} \cdot Q(n-\mu+1) \cdots Q(n) .
$$

For $\mu \geq p$ the product $Q(n-\mu+1) \ldots Q(n)$ has at least $p$ factors. By the assumption $(26)$ that the polynomial $Q$ "splits completely" $(\bmod p)$ in $q$ linear factors of the form $a x+b, p \nmid a$, one gets

$$
p^{q} \mid Q(n-\mu+1) \cdots Q(n), \mu \geq p .
$$

If one remembers that the $\alpha_{j}, 1 \leq j \leq h$, could be chosen as integers (compare remark ii) to theorem 1) one recognizes from (4) and (28) that the coefficient $\gamma_{n} k_{n}$ of the starting polynomial $P$ is also an integer. Therefore we have from (37) and (38)

$$
p^{q} \mid \gamma_{n} \cdot k_{n-\mu}, \quad p \leq \mu \leq n \leq m .
$$

From this we conclude with (36)

$$
p^{q} \mid P_{\mu}^{(\rho)}\left(\alpha_{j}\right), p \leq \mu \leq m, 0 \leq \rho<q, 1 \leq q, 0 \leq j \leq h\left(\alpha_{0}:=0\right)
$$

\section{The values $P^{*(\nu)}\left(\alpha_{j}\right)$ and the non-vanishing \\ of the linear form $\Lambda^{*}$}

We apply the divisibility properties proved in chapter 5 to show that the linear form $\Lambda^{*}$ of $\left(25_{1}\right)$

$$
\Lambda^{*}:=s_{0} P^{*}(0)+\sum_{j=1}^{h} \sum_{\nu=0}^{\nu_{0}-1} s_{j}^{(\nu)} P^{*(\nu)}\left(\alpha_{j}\right)+\sum_{j=1}^{j_{0}} s_{j}^{\left(\nu_{0}\right)} P^{*\left(\nu_{0}\right)}\left(\alpha_{j}\right)
$$

does not vanish. $P^{*}$ (compare (6)) is defined with the polynomial $P$ of (28) by

$$
P^{*}(x)=\sum_{\mu=0}^{m} P_{\mu}(x), m=p \cdot q \cdot h+(p-1) q+\nu_{0}=: \operatorname{deg} P
$$


It follows from (5) and (30)

$$
P^{*}(0)=\sum_{\mu=p q}^{m} P_{\mu}(0)=\sum_{\mu=p q}^{m} \gamma_{\mu}
$$

and from (32) and (35)

$$
P^{*(\rho)}\left(\alpha_{j}\right)=\sum_{\mu=p}^{m} P_{\mu}^{(\rho)}\left(\alpha_{j}\right) j \neq j_{0}, \rho \leq q-1,
$$

$(43)$

$$
P^{*(\rho)}\left(\alpha_{j_{0}}\right)=\sum_{\mu=p}^{m} P_{\mu}^{(\rho)}\left(\alpha_{j_{0}}\right), \rho<\nu_{0} .
$$

With (40) we get from (43)

$$
p^{q} \mid P^{*(\rho)}\left(\alpha_{j}\right)\left(j \neq j_{0}, \rho \leq q-1\right) \quad \text { and } \quad p^{q} \mid P^{*(\rho)}\left(\alpha_{j_{0}}\right)\left(\rho<\nu_{0}\right) .
$$

Therefore we have

$$
p^{q} \mid\left(s_{0} P^{*}(0)+\sum_{j=1}^{h} \sum_{\rho=0}^{\nu_{0}-1} s_{j}^{(\rho)} P^{*(\rho)}\left(\alpha_{j}\right)+\sum_{j=1}^{j_{0}-1} s_{j}^{\left(\nu_{0}\right)} P^{*\left(\nu_{0}\right)}\left(\alpha_{j}\right)\right)
$$

which is the same (compare the definition of $\Lambda^{*}$ in $\left(25_{1}\right)$ ) as

$$
p^{q} \mid\left(\Lambda^{*}-s_{j_{0}}^{\left(\nu_{0}\right)} P^{*\left(\nu_{0}\right)}\left(\alpha_{j_{0}}\right)\right) .
$$

From (32) we get

$$
P^{*\left(\nu_{0}\right)}\left(\alpha_{j_{0}}\right)=P_{p-1}^{\left(\nu_{0}\right)}\left(\alpha_{j_{0}}\right)+\sum_{\mu=p}^{m} P_{\mu}^{\left(\nu_{0}\right)}\left(\alpha_{j_{0}}\right) .
$$

Because of (40) the second term on the right of (46) is a multiple of $p^{q}$ whereas the first term is not divisible by $p^{q}$ (see (34)). Therefore we get from (46)

$$
p^{q} \times P^{*\left(\nu_{0}\right)}\left(\alpha_{j_{0}}\right), q \geq 1 .
$$

Since we assumed in (27) $p \nmid s_{j_{0}}^{\left(\nu_{0}\right)}$ we get with (47)

$$
p^{q} \backslash s_{j_{0}}^{\left(\nu_{0}\right)} P^{*\left(\nu_{0}\right)}\left(\alpha_{j_{0}}\right) .
$$

Together with (45) one derives now the important relation

$$
p^{q} \backslash \Lambda^{*} \text { or } \Lambda^{*} \neq 0 .
$$




\section{A lower bound for $\Lambda^{*}$}

Let $t$ be a prime number and let $\mu_{t}$ denote the number

$$
\begin{array}{r}
\mu_{t}:=|\{x \in \mathbb{Z}, 0 \leq x<t, Q(x) \equiv 0(\bmod t)\}| ; \\
|\{\ldots\}| \text { denotes the cardinality of the set }\{\ldots\} .
\end{array}
$$

After Nagell [9] one has the estimate

$$
\sum_{t \leq x} \mu_{t} \frac{\log t}{t}=\kappa \log x+\mathrm{O}(1)
$$

where $\kappa$ denotes the number of irreducible factors of $Q$.

Let $p \in \mathbb{P}$ be the prime number which was chosen in Chapter 5 (compare (26), (27)). In (37) we have seen that for $\mu \geq p$ every coefficient $\gamma_{n} k_{n-\mu}$ in the representation $(36)$ of $P_{\mu}^{(\rho)}\left(\alpha_{j}\right)$ contains the factor

$$
Q(n-\mu+1) \ldots Q(n)(p \leq \mu \leq n \leq m) .
$$

In all these products the argument of $Q$ goes at least through $p$ consecutive positive integers. Therefore, using the definition of $\mu_{t}$ in (49), in each one of these products the prime number $t$ occurs at least in order $\mu_{t}\left[\frac{p}{t}\right]$. In consequence for $\mu \geq p$, every number $P_{\mu}^{(\rho)}\left(\alpha_{j}\right)$ is divisible by a positive integer $B_{p}$ with

$$
B_{p} \geq \prod_{t \in \mathbb{P}} t^{\mu_{t}\left[\frac{p}{t}\right]} .
$$

Regarding the construction of $P^{*}$ (compare (41)) one has by(43)

$$
B_{p}\left|P^{*(\rho)}\left(\alpha_{j}\right), j \neq j_{0}, 0 \leq \rho<q, B_{p}\right| P^{*(\rho)}\left(\alpha_{j_{0}}\right), 0 \leq \rho<\nu_{0} .
$$

The same divisibility property can be proved for $\alpha_{0}:=0$ so one has

$$
B_{p} \mid P^{*}(0) \text {. }
$$

For $j=j_{0}$ and $\nu=\nu_{0}$ it was shown in (46)

$$
P^{*\left(\nu_{0}\right)}\left(\alpha_{j_{0}}\right)=P_{p-1}^{\left(\nu_{0}\right)}\left(\alpha_{j_{0}}\right)+\sum_{\mu=p}^{m} P_{\mu}^{\left(\nu_{0}\right)}\left(\alpha_{j_{0}}\right) .
$$

The second term on the right is by the argument above again divisible by $B_{p}$. For the first term one gets by (32) and (33)

$$
\left((p-1) q+\nu_{0}\right) ! \mid P_{p-1}^{\left(\nu_{0}\right)}\left(\alpha_{j_{0}}\right)
$$


From the relations (49) - (54) we conclude that

$$
\begin{aligned}
\Lambda^{*}=\left(s_{0} P^{*}(0)+\sum_{j=1}^{h} \sum_{\rho=0}^{\nu_{0}-1} s_{j}^{(\rho)} P^{*(\rho)}\left(\alpha_{j}\right)+\sum_{j=1}^{j_{0}-1} s_{j}^{\left(\nu_{0}\right)} P^{*\left(\nu_{0}\right)}\left(\alpha_{j}\right)\right) & \\
& +s_{j_{0}}^{\left(\nu_{0}\right)} P^{*\left(\nu_{0}\right)}\left(\alpha_{j_{0}}\right)
\end{aligned}
$$

can be divided by

$$
D:=\text { g.c.d. }\left(\left((p-1) q+\nu_{0}\right) !, \prod_{t \in \mathbb{P}} t^{\mu_{t}\left[\frac{p}{t}\right]}\right)
$$

that means

$$
D \mid \Lambda^{*}
$$

One has

$$
D \geq \text { g.c.d. }\left((p-1) !, \prod_{t \in \mathbb{P}} t^{\mu_{t}\left[\frac{p}{t}\right]}\right) \geq \text { g.c.d. }\left(\prod_{t \in \mathbb{P}} t^{\left[\frac{p-1}{t}\right]}, \prod_{t \in \mathbb{P}} t^{\mu_{t}\left[\frac{p-1}{t}\right]}\right) .
$$

This gives, because of $\mu_{t} \leq q$,

$$
D \geq \exp \left(\frac{1}{q} \sum_{t \leq p} \mu_{t} \frac{\log t}{t} \cdot t \cdot\left[\frac{p-1}{t}\right]\right) .
$$

Finally one gets from (50), (56) and (57) the lower bound

$$
\left|\Lambda^{*}\right| \geq|D| \geq \exp \left(\frac{\kappa}{q} p \log p+\mathrm{O}(p)\right) .
$$

\section{An estimate of the remainder terms $\Delta^{(\nu)}\left(\alpha_{j}\right)$}

$\Delta(x)$ was defined in (10). Since we have $\gamma_{n}=0$ for $0 \leq n<q \cdot p$, we get for $0 \leq \nu \leq q-1$

$$
\begin{aligned}
\Delta^{(\nu)}(x) & =-\sum_{n=q \cdot p}^{m} \gamma_{n} \cdot \sum_{\rho=n+1}^{\infty} k_{\rho} \nu !\left(\begin{array}{l}
\rho \\
\nu
\end{array}\right) x^{\rho-\nu} \\
& =-\sum_{n=q \cdot p}^{m} \gamma_{n} \cdot k_{n} \nu !\left(\begin{array}{l}
n \\
\nu
\end{array}\right) x^{n-\nu} \cdot \sum_{\rho=n+1}^{\infty} \frac{\left(\begin{array}{l}
\rho \\
\nu
\end{array}\right)}{\left(\begin{array}{l}
n \\
\nu
\end{array}\right)} \frac{k_{\rho}}{k_{n}} \cdot x^{\rho-n} .
\end{aligned}
$$

We have $Q \in \mathbb{Z}[x]$ and $Q$ is of degree $q$. Therefore exists an integer $n_{0}(Q)$ so that

$$
\text { for all } n>n_{0}(Q),|Q(n)| \geq \frac{n^{q}}{2} \text {. }
$$

In (59) we have $\rho>n \geq q p$. From (60) follows that if $p$ is sufficiently large we have

$$
\left|\frac{k_{\rho}}{k_{n}}\right|=\frac{1}{|Q(n+1) \ldots Q(\rho)|} \leq\left(\frac{2}{n^{q}}\right)^{(\rho-n)} .
$$


Since we can take the prime number $p$, chosen in (26) and (27), as large as we want, we can consider only numbers $x$ with the property

$$
\frac{4|x|}{p}<\frac{1}{2} \text {. }
$$

Then one gets the estimate

$$
\begin{aligned}
\left|\sum_{\rho=n+1}^{\infty} \frac{\left(\begin{array}{l}
\rho \\
\nu
\end{array}\right)}{\left(\begin{array}{l}
n \\
\nu
\end{array}\right)} \frac{k_{\rho}}{k_{n}} x^{\rho-n}\right| & \leq \sum_{\rho=n+1}^{\infty} 2^{\rho}\left(\left(\frac{2}{n^{q}}\right)|x|\right)^{\rho-n} \\
& =\sum_{\rho=n+1}^{\infty} 2^{n}\left(\frac{4}{n^{q}}|x|\right)^{\rho-n} \\
& \leq 2^{n+3} \cdot \frac{|x|}{n^{q}} \leq 2^{n+2}|x| .
\end{aligned}
$$

Let $P_{*}$ denote the polynomial

$$
P_{*}(x):=\sum_{n=q \cdot p}^{m}\left|\gamma_{n} k_{n}\right| x^{n} .
$$

With (59) and (63) follows

$$
\left|\Delta^{(\nu)}(x)\right| \leq 4 \cdot 2^{m}|x| P_{*}^{(\nu)}(|x|), 0 \leq \nu \leq q-1,|x|<\frac{p}{8} .
$$

If $\tilde{p}$ and $\tilde{q} \in \mathbb{C}[x]$ are polynomials,

$$
\tilde{p}(x):=\sum_{\ell=0}^{L} a_{\ell} x^{\ell}, \tilde{q}(x):=\sum_{\ell=0}^{L} b_{\ell} x^{\ell},
$$

one writes

$$
\tilde{p}(x) \prec \tilde{q}(x): \Leftrightarrow \text { for all } \ell, 0 \leq \ell \leq L,\left|a_{\ell}\right| \leq\left|b_{\ell}\right| .
$$

From the definition of $P$ in (4) and (28) it follows that there are constants $c_{1}>0$ and $c_{2}>0$, which depend only on $\alpha_{1}, \ldots, \alpha_{h}$ so that we have

$$
P_{*}(x) \prec\left(c_{1}(1+x)\right)^{m}
$$

and

$$
P_{*}^{(\nu)}(x) \prec c_{2}^{m}(1+x)^{m}, 1 \leq \nu \leq q-1 .
$$

For a $p \in \mathbb{P}$ that satisfies

$$
8\left|\alpha_{j}\right| \leq p, 1 \leq j \leq h
$$

we get from (65) and (68)

$$
\left|\Delta^{(\nu)}\left(a_{j}\right)\right| \leq c_{3}^{p}, 1 \leq j \leq h .
$$

Here $c_{3}>0$ is a constant, which depends on $\alpha_{1}, \ldots, \alpha_{h}$ and $Q$, but not on $p \in \mathbb{P}$. 


\section{Proof of the linear independence over $\mathbb{Q}$ of the numbers}

$$
1, G^{(\nu)}\left(\alpha_{j}\right)(1 \leq j \leq h, 0 \leq \nu \leq q-1)
$$

We have to show that for every non-zero vector in (11) the linear form

$$
\Lambda:=s_{0} G(0)+\sum_{\nu=0}^{q-1} \sum_{j=1}^{h} s_{j}^{(\nu)} G^{(\nu)}\left(\alpha_{j}\right), G(0)=1
$$

is different from zero.

In (14) we have seen that the linear forms $\Lambda$ and $\Lambda^{*}$ are connected in the following way:

$$
\Lambda^{*}=P^{*}(0) \Lambda+\sum_{\nu=0}^{q-1} \sum_{j=1}^{h} s_{j}^{(\nu)} \Delta^{(\nu)}\left(\alpha_{j}\right) .
$$

If $H$ denotes the height of the vector in (11),

$$
H:=\max _{\nu, j}\left(\left|s_{j}^{(\nu)}\right|\right),
$$

it follows from (72) with (58) and (70)

$$
\begin{aligned}
\left|P^{*}(0) \Lambda\right| & \geq\left|\Lambda^{*}\right|-\sum_{\nu=0}^{q-1} \sum_{j=1}^{h}\left|s_{j}^{(\nu)} \Delta^{(\nu)}\left(\alpha_{j}\right)\right| \\
& \geq \exp \left(\frac{\kappa}{q} p \log p+\mathrm{O}(p)\right)-H \exp \left(p \cdot c_{4}\right)
\end{aligned}
$$

where $c_{4}>0$ is a constant independent of $p$ and $H$. From (74) one gets for sufficiently large $p$ (depending on $H$ )

$$
\Lambda \neq 0,
$$

which proves the linear independence over $\mathbb{Q}$ of the numbers $1, G\left(\alpha_{1}\right), \ldots$, $G^{(q-1)}\left(\alpha_{h}\right)$.

\section{References}

[1] P. Bundschuh, R. Wallisser, Maße für die lineare Unabhängigkeit von Werten ganz transzendenter Lösungen gewisser Funktionalgleichungen I Bd. 69 (1999) II Bd. 73 (2003). Abh. Math Sem. Univ. Hamburg.

[2] F. CARLSON, Sur une propriété arithmétique de quelques fonctions entières. Arkiv för Mathematik, Astronomi och Fysik. Bd 25A. N: 07 (1935).

[3] R. DEDEKIND, Über den Zusammenhang zwischen der Theorie der Ideale und der höheren Kongruenzen, Abh. Akad. Wiss. Göttingen 23 (1878), 3-37.

[4] N.I. Fel'dman, Yu.V. Nesterenko, Transcendental Numbers, Number Theory IV. Encycl. of Math. Sc. 44 (1998), Springer.

[5] I. Gerst, J. Brillhart, On the prime divisors of polynomials. Amer. Math. Monthly 78 (1971).

[6] H. HAsse, Bericht über neuere Untersuchungen und Probleme aus der Theorie der algebraischen Zahlkörper, 2nd ed., Physica, Würzburg, 1965.

[7] D.Hilbert, Über die Transcendenz der Zahlen e und $\pi$. Math. Ann. 43 (1893), 216-219.

[8] A. Hurwitz, Beweis der Transzendenz der Zahl e. Math. Ann. 43 (1893), 220-221. 
[9] T. NAgell, Généralisation d'un théorème de Tchebycheff. Journ. de Math. ( $8^{e}$ serie), tome IV (1921).

[10] O. Perron, Irrationalzahlen. Chelsea, New York, (1951).

[11] I. SchUR, Über die Existenz unendlich vieler Primzahlen in einigen speziellen arithmetischen Progressionen. S.-B. Berlin. Math. Ges. 11 (1912), 40-50.

[12] Тн. SкоLem, Some theorems on irrationality and linear independence. Skand. Mat. Kongr. 11, Trondheim 1949, 77-98.

Rolf WALLISSER

Mathematisches Institut der Universität Freiburg

Eckerstr.1

79104 Freiburg, Deutschland

E-mail : rowa@sun1.mathematik.uni-freiburg.de 